\title{
Next-generation air-navigation displays
}

Dieter Cuypers, Herbert De Smet, Xavier Hugel, and Guilhem Dubroca

Future airplanes could be fitted with several short-throw wide-angle LED projectors to create a seamless tiled cockpit interactive airnavigation display.

Aircraft currently use several rugged liquid-crystal displays in the cockpit to visualize flight data. As these displays get larger and the number of separate screens is reduced, the cockpit workspace becomes better integrated. Taking rationalization of air-navigation equipment to the next level implies a highly integrated single interactive display to assist in all tasks in the cockpit, including navigation, flight-preparation documentation, display of airport maps, non-flight-related functions, and external camera views. This type of display would consist of a reconfigurable dashboard, designed to show important visual elements in prominent view, according to need. Adding a touchscreen command on the whole surface of the display would provide a complete, self-contained means of controlling the cockpit (see Figure 1). Ultimately, this solution would substantially improve the human-machine interface. ${ }^{1}$

Making a single large display with a curved surface spreading across an odd shape is a key technological challenge. Until now, other niche applications, such as simulation and control environments, scientific visualization, and gaming/virtual reality, have adopted solutions based on tiling individual liquid-crystal panels or emissive displays (including field-emission displays, plasma panel displays, and organic LEDs). This approach, however, does not lead to a truly seamless screen. To devise a solution to this challenge, further constraints specific to the aircraft industry and related to the level of reliability and safety requirements need to be taken into account. In addition, new developments must match existing solutions in terms of space constraints. Here we report our work on projection-imaging technology to simultaneously tackle all the issues related to form, seams, and space constraints.

The main appeal of projection-imaging technology devices is their ability to modulate light by relying on either switchable

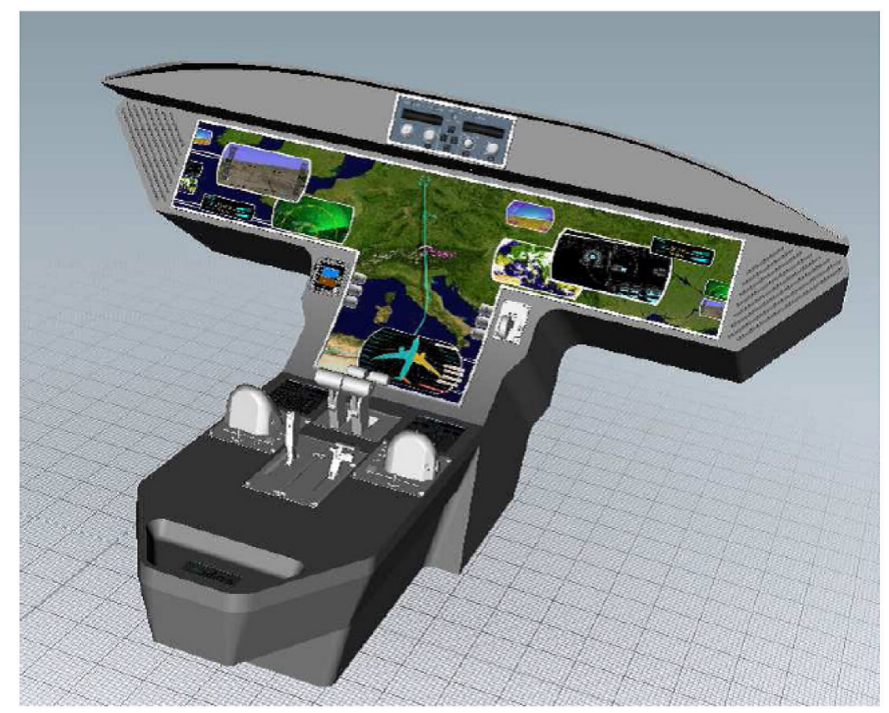

Figure 1. Artist's rendering of a single-display interactive cockpit. (Courtesy of Thales Avionics.)

digital micromirror devices (DMDs) or liquid-crystal-on-silicon (LCOS) panels. We chose the latter because of their ruggedness during flight, their large range of resolution, and their availability from multiple manufacturers. Guided by the need to eliminate the risk of any color artifacts and to ensure the highest reliability, we opted for a three-channel color system.

Conventional projection systems typically use arc lamps as a light source. However, they are ill suited to the avionic environment owing to risk of explosion and fire, and they are not considered reliable. We therefore opted for a custom-designed illumination system that employs high-luminance LEDs. Although they are ubiquitous in many illumination applications, they are not an obvious choice for the aircraft industry because of the high demands on brightness imposed by the stringent need for screen legibility in direct sunlight. We were able to meet this requirement by using state-of-the-art projection LEDs combined with a carefully designed light-management system. Moreover,

Continued on next page 


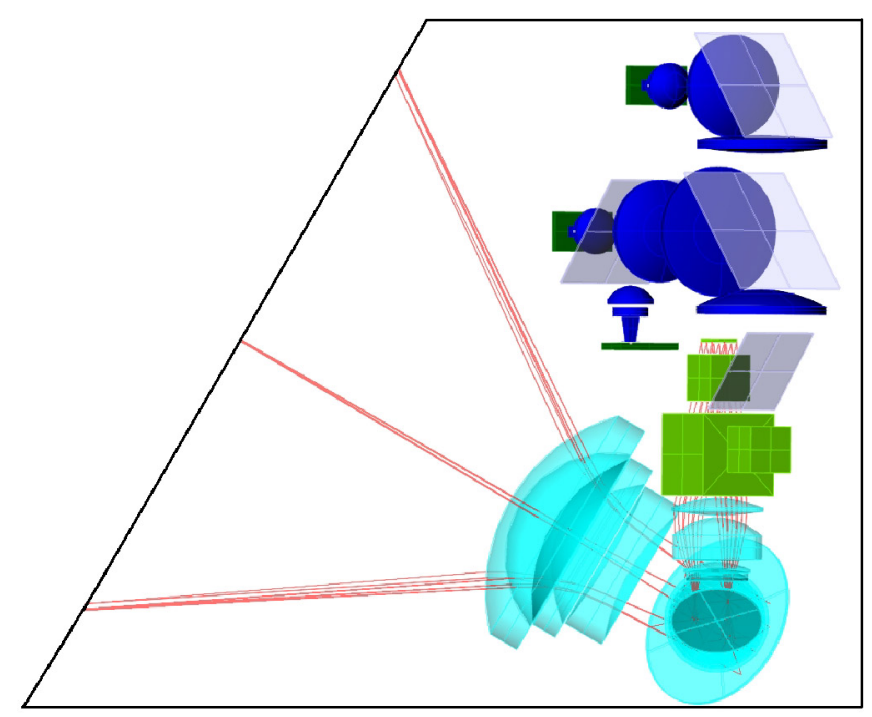

Figure 2. Design of the short-throw ultra-wide-angle projector.

LEDs presented the added benefit of being highly reliable over a long period of time-subject to tight control of the LED's junction temperature with an adequate cooling system-thus meeting aircraft constraints. The LEDs are selected to emphasize luminance over electrical efficiency and matched with collectionoptics parts designed to minimize further losses in the luminous flux. The lens system and projection screen design are equally matched to each other to ensure preservation of the luminance. The screen itself maximizes the light output in the specified viewing cone of the crew members.

One of the biggest challenges of this project was to fit projectors in the cockpit without taking up more space than the current LCD display units. This meant that only $25 \mathrm{~cm}$ was available to fit the entire optical assembly, with an even shorter throw distance. To avoid compromising image quality, we designed a dedicated short-throw ultra-wide-angle projection lens to accommodate this short-range projection while keeping a small footprint ${ }^{2}$ (see Figure 2).

We then addressed the need to maintain a high contrast ratio even at high levels of ambient light by using a black-matrix screen. Its performance was subject to the provision of a wellcollimated incident beam that was guaranteed by adding a film with a Fresnel lens to the screen stack to enhance brightness uniformity.

Given that it is not possible to use a single projector to cover the cockpit's entire curvature and special T-shape form, we split up the overall display area in several sub-projection areas, each with their own projector unit. This required that the Fresnel lenses be linear, so that the individual projection images could

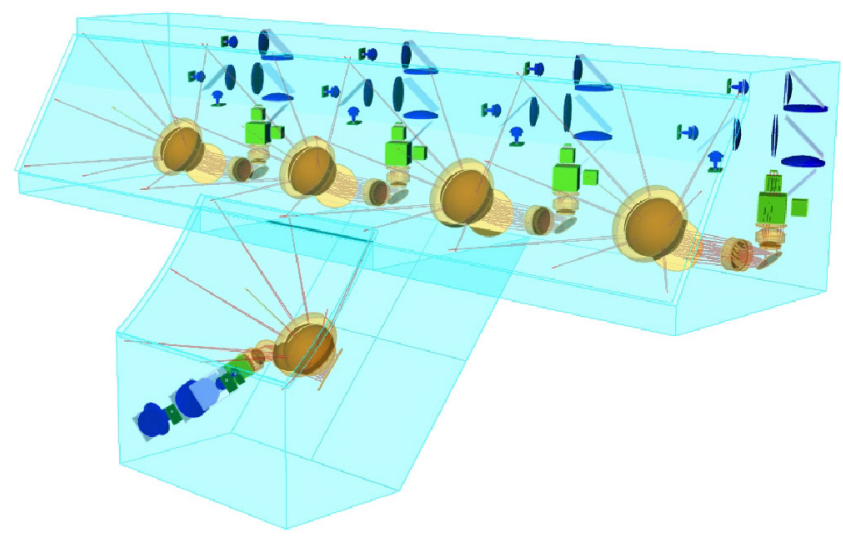

Figure 3. Arrangement of five wide-angle projectors that make up a single seamless display in the cockpit.

be tiled through blending techniques to form one large seamless display (see Figure 3).

At the time of writing, the prototype of a single seamless cockpit display intended to demonstrate the capabilities of projection technology was nearing completion. Touch screen input was to be provided by a proprietary optical design that does not alter the readability of the display. The next step will involve evaluating the system both from a technical and a human-factor viewpoint.

The work described in this paper was realized with support from the European Commission under the Seventh Framework Programme, Theme Transports, project ODICIS, grant ACP8-GA-2009-233605. The authors would like to acknowledge all the partners of this project for their contribution.

\section{Author Information}

\section{Dieter Cuypers}

Imec - Ghent University

Zwijnaarde, Belgium

Dieter Cuypers obtained his $\mathrm{PhD}$ from Ghent University. Since then, he has been working in the Department of Electronics and Information Systems of the same university and collaborating with the nanoelectronics research company imec. He focuses mainly on visualization technology-specifically microdisplays - and has interests in other areas, including optics and thin-film processing. He is a member of the Society for 
Information Display (SID) and SPIE and the author or co-author of over 40 papers.

\section{Herbert De Smet}

CMST

Ghent University

Zwijnaarde, Belgium

Herbert De Smet obtained his $\mathrm{PhD}$ in integrated drivers for flat-panel displays from Ghent University (1994). Since 1995, he has been working for imec, in charge of several European and national research projects concerning displays and microdisplays. In 2000, he also became a professor at Ghent University, where he teaches courses in the field of microsystems, sensors, and actuators. He is the secretary of the Mid-Europe chapter of SID, the associate editor for the Journal of Information Display, and the author or co-author of more than 160 papers.

\section{Xavier Hugel and Guilhem Dubroca}

Optinvent

Rennes, France

References

1. http:/ / www.odicis.org One Display for a Cockpit Interactive Solution. Accessed 16 June 2011.

2. D. Cuypers, H. D. Smet, X. Hugel, G. Dubroca, and A. Van Calster, Projection displays in avionics, 17th Int'1 Display Workshops, pp. 1437-1440, 2010. 